CALT-68-1953

DOE RESEARCH AND

DEVELOPMENT REPORT

hep-th/9409158

\title{
QUANTUM CORRECTIONS TO BLACK HOLE ENTROPY IN STRING THEORY
}

\author{
Atish DABHOLKaR \\ Lauritsen Laboratory of High Energy Physics \\ California Institute of Technology \\ Padadena, CA 91125, USA \\ e-mail: atish@theory.caltech.edu
}

\begin{abstract}
The one-loop contribution to the entropy of a black hole from field modes near the horizon is computed in string theory. It is modular invariant and ultraviolet finite. There is an infrared divergence that signifies an instability near the event horizon of the black hole. It is due to the exponential growth of the density of states and the associated Hagedorn transition characteristic of string theory. It is argued that this divergence is indicative of a tree level contribution, and the Bekenstein-Hawking-Gibbons formula for the entropy should be understood in terms of string states stuck near the horizon.
\end{abstract}

$9 / 94$ 


\section{Introduction}

A major puzzle in the physics of black holes concerns the interpretation of the entropy associated with a black hole. In the semi-classical approximation, the entropy is given by the Bekenstein-Hawking formula [1,2]:

$$
S_{B H}=\frac{A}{4 G \hbar},
$$

where A is the area of the event horizon of the black hole and $G$ is Newton's constant. This remarkable formula points to a deep connection between quantum mechanics, gravity and thermodynamics. However, its relation to statistical mechanics is largely mysterious. Gibbons and Hawking [3] have obtained the same expression from the quantum partition function for gravity. Even so, a satisfactory statistical interpretation of the entropy in terms of enumeration of states is lacking.

Another related puzzle concerns the entropy of quantum fluctuations seen by the Schwarzschild observer. As pointed out by 't Hooft [4], the contribution to the entropy coming from the field modes very near the horizon is ultraviolet divergent. Several authors have found a similar divergence in Rindler spacetime which approximates the geometry of a large black hole very near the horizon [5, 6, 7, 8, 9, 10]. There is a simple description of the leading divergence. A fiducial observer that is stationed at a fixed radial distance from the black hole, has to accelerate with respect to the freely falling observer in order not to fall into the black hole. Very near the horizon, the fiducial observer is like a Rindler observer in flat Minkowski space. As a result, she sees a thermal bath [11] at a position-dependent proper temperature $T(z)=\frac{1}{2 \pi z}$ where $z$ is the proper distance from the horizon. Using Planck's formula for a single massless boson we get the entropy density:

$$
s(z)=\frac{4}{3} \frac{\pi^{2}}{30}\left(\frac{1}{2 \pi z}\right)^{3} .
$$

Note that we have been able to define the entropy density because entropy is an extensive quantity as it should be. However, the dominant contribution comes from the region near the horizon $z=0$ and is not extensive but proportional to the area. If we put a cutoff on

the proper distance at $z=\epsilon$ (or alternatively on proper temperature) the total entropy is:

$$
\begin{aligned}
S & =\int_{\epsilon}^{\infty} s(z) A d z \\
& =\frac{A}{360 \pi \epsilon^{2}},
\end{aligned}
$$


where $A$ is the area in the transverse dimensions. This is in agreement with the result obtained in 4.5 by other means. Because the thermal bath is obtained by tracing over states that are not accessible to the observer in the Rindler wedge, this entropy is also the same as the 'entropy of entanglement' [8,6,10, 12] or the 'geometric entropy' [7, 9]. For a massive particle of mass $m$ there will be corrections to this formula which will be down by powers of $m \epsilon$.

't Hooft has advocated that the entropy of entanglement should account for the black hole entropy. This would offer a statistical interpretation of the black hole entropy and also a natural explanation for the area dependence. In the context of field theory, there are several difficulties with this appealing idea. For example, the entropy of entanglement is divergent and depends on an arbitrary ultraviolet cutoff whereas the black hole entropy is finite and depends on the Planck length. The entropy of entanglement depends on the species and the couplings of the various particles in the theory whereas the black hole entropy is species-independent. Finally, it is difficult to see how the entropy of entanglement, which always starts at one loop can possibly account for the black hole entropy which is inversely proportional to the coupling constant. 't Hooft has argued that it is necessary to understand the ultraviolet structure of the theory in order to address these issues. He has conjectured that these difficulties will be resolved once the correct short distance structure is known. He has further suggested that this divergence of entropy in field theory is intimately related to the puzzle of loss of information in black hole evaporation. If the entropy does have a statistical interpretation in terms of counting of states, then its divergence would suggest an infinite number of states associated with a finite mass black hole. As long as the black hole has an event horizon, it can apparently store an arbitrary amount of information in terms of correlations between the outgoing radiation and the high energy modes near the horizon. When the horizon eventually disappears, the information in these correlations is irretrievably lost.

This conjecture could hardly be tested in field theory especially when we are dealing with a non-renormalizable theory such as quantum gravity. Fortunately, string theory offers a suitable framework for addressing this question. It is a perturbatively finite theory of quantum gravity and comes with a well-defined matter content. Moreover, Susskind 
[13,14] has argued that string theory may also possess some of the properties required for describing black hole evaporation without information loss. It is therefore of great interest to know how the ultraviolet behavior of the entropy is controlled in string theory. Until now, the finiteness of string theory has been little utilized in quantum gravity. The main obstacle has been that many of the difficult questions in quantum gravity require a non-perturbative formalism of the theory. By contrast, the question about the ultraviolet finiteness of entropy is accessible entirely within perturbation theory. One can hope that string theory will illuminate this question in important ways.

In the next section, we compute the one-loop contribution to the entropy of a very large black hole in the twenty-six dimensional bosonic string. We obtain a modular invariant expression which easily generalizes to higher loops. The generalization to superstrings is also straightforward. The main result of this paper is that the quantum contributions to the entropy of a very large black hole in string theory are indeed finite in the ultraviolet. This finiteness comes about by a mechanism well-known in string theory. In field theory, the divergence comes from summing over small loops in which the virtual particles travel for a very short proper time. In string theory, this region of short proper time belongs to certain corners of the parameter space that is excluded by modular invariance.

It turns out that the entropy, even though finite in the ultraviolet, is divergent in the infrared. The divergence is due to a tachyonic instability very near the horizon and most likely is associated with the Hagedorn phase transition [15]. Recall that the fiducial observer sees a hot thermal bath near the horizon. Moreover, the density of states at high energy has an exponential growth characteristic of string theory. As a result, beyond a certain temperature-the so-called Hagedorn temperature-the thermal ensemble is rendered unstable. If there is a phase transition and a condensate is formed, then the latent heat of the transition can induce a tree level contribution to the entropy [16]. Thus, it seems plausible that we can understand the dependence of the entropy on coupling constant. Moreover, if the contribution to the entropy comes from the fundamental degrees of freedom of string theory, then quite possibly it is independent of the species of particles in the low energy theory. Finally, as we shall see, in the case of black holes, this condensate will be restricted very close to the horizon and will extend only in the transverse directions. There 
can also be an additional contribution to the entropy from the string states with endpoints immersed in the 'deconfined' soup of strings very near the horizon [14]. This raises the exciting possibility that the area dependence of the tree level Bekenstein-Hawking formula for the entropy can be understood in terms of statistical mechanics.

\section{Black Hole Entropy in the Bosonic String at One Loop}

In this section we derive the expression for the entropy in the bosonic string theory at one loop. This derivation is essentially an application of equations (1.2) and (1.3). These considerations are also relevant to Rindler strings. Some aspects of Rindler strings have been discussed earlier in [17].

As our starting point we take the expression for the entropy in field theory in the proper time formalism. The reasoning leading to the corresponding expression in string theory is similar to the one employed in the derivation of the cosmological constant [18]. Let us consider the free-energy density for a single boson of mass $m$ at finite temperature $\beta^{-1}$ :

$$
f\left(\beta, m^{2}\right)=\frac{1}{\beta} \int \frac{d^{d-1} k}{(2 \pi)^{d-1}} \log \left(1-e^{-\beta \omega_{k}}\right) .
$$

To write it in the proper time formalism we first introduce $1=\int_{0}^{\infty} 2 \omega \int_{-i \infty}^{i \infty} \frac{d s}{2 \pi i} e^{s\left(\omega^{2}-\omega_{k}^{2}\right)}$, expand the logarithm and then perform the gaussian momentum integrals to obtain [19,20]

$$
f\left(\beta, m^{2}\right)=-\int_{0}^{\infty} \frac{d s}{s} \frac{1}{(2 \pi s)^{d / 2}} \sum_{r=1}^{\infty} e^{-m^{2} s / 2-r^{2} \beta^{2} / 2 s} .
$$

The entropy density is as usual $s\left(\beta, m^{2}\right)=\beta^{2} \frac{\partial f}{\partial \beta}$. For Rindler observers we simply put $\beta=2 \pi z$ to obtain the local entropy density and then integrate as in (1.3) to get the total entropy:

$$
S\left(m^{2}\right)=A \int_{0}^{\infty} d z(2 \pi z)^{3} \int_{0}^{\infty} \frac{d s}{s^{2}} \frac{1}{(2 \pi s)^{d / 2}} \sum_{r=1}^{\infty} r^{2} e^{-m^{2} s / 2-2 \pi^{2} r^{2} z^{2} / s} .
$$

There is an ultraviolet divergence as in (1.3) because the $s$ integral diverges near $s=0$ for small $z$. Notice that we have to be careful while interchanging the order of integration because the integral over $s$ is not uniformly convergent as a function of $z$. We can interchange the order by putting appropriate cutoffs for both the integrals. 
It is straightforward to generalize these formulae to the spectrum of the bosonic string in $d=26$ by summing the expression (2.2) over $m^{2}$. In the light cone gauge the spectrum is given in terms of the occupation numbers of the right-moving and the left-moving oscillators $N_{n i}$ and $\tilde{N}_{n i}[21]$ :

$$
m^{2}=\frac{2}{\alpha^{\prime}}\left[-2+\sum_{i=1}^{24} \sum_{n=1}^{\infty} n\left(N_{n i}+\tilde{N}_{n i}\right)\right]
$$

subject to the constraint

$$
\sum_{i=1}^{24} \sum_{n=1}^{\infty} n\left(N_{n i}-\tilde{N}_{n i}\right)=0
$$

The constraint can be enforced by introducing

$$
\int_{-\frac{1}{2}}^{\frac{1}{2}} d \theta e^{2 \pi i n \theta\left(N_{n i}-\tilde{N}_{n i}\right)}
$$

into the sum. It is convenient to introduce a complex variable $\tau=\theta+i \frac{s}{2 \pi \alpha^{\prime}}$. The sum can then be easily performed to obtain

$$
f(\beta)=-\frac{1}{2}\left(\frac{1}{4 \pi^{2} \alpha^{\prime}}\right)^{-13} \int_{\mathcal{S}} \frac{d^{2} \tau}{\operatorname{Im} \tau^{2}}(\operatorname{Im} \tau)^{-12}\left|\eta\left(e^{2 \pi i \tau}\right)\right|^{-48} \sum_{r=1}^{\infty} \exp -\frac{r^{2} \beta^{2}}{4 \pi \alpha^{\prime} \operatorname{Im} \tau} .
$$

Here $\eta$ is the Dedekind eta function,

$$
\eta(q)=q^{\frac{1}{24}} \prod_{n=1}^{\infty}\left(1-q^{n}\right)
$$

and the region of integration is the $\operatorname{strip} \mathcal{S}$

$$
-\frac{1}{2} \leq \operatorname{Re} \tau \leq \frac{1}{2}, \quad 0 \leq \operatorname{Im} \tau \leq \infty
$$

The total entropy computed from this formula has a divergence for each mode coming from the region near $\operatorname{Im} \tau=0$.

This would be the end of the story if we were dealing with a field theory of the string modes. But string theory is not merely a sum of field theories because of duality [19]. The sum of field theories overcounts the correct string answer. For example, a four point amplitude is represented by a single string diagram but gives rise to two separate diagrams in the $s$ and the $t$ channel in field theory. The correct generalization of the above formulae 
to string theory is more subtle and requires a proper treatment of this overcounting. We do this by noting that using a modular transformation of $\tau$, every point in $\mathcal{S}$ can be mapped onto the fundamental domain $\mathcal{F}$ of a torus [20]:

$$
|\tau|>1,-\frac{1}{2}<\operatorname{Re} \tau<\frac{1}{2}, \operatorname{Im} \tau>0
$$

Recall that the modular group $\Gamma$ at one-loop is the group of disconnected diffeomorphisms of a torus up to conformal equivalences. It is isomorphic to the group $S L(2, \mathbb{Z}) / Z_{2}$ under which $\tau$ transforms as

$$
\tau^{\prime}=\frac{a \tau+b}{c \tau+d}, \quad\left(\begin{array}{ll}
a & b \\
c & d
\end{array}\right) \in S L(2, \mathbb{Z}) .
$$

We have to divide the $S L(2, \mathbb{Z})$ by $Z_{2}$ because the elements $\{\mathbf{I},-\mathbf{I}\}$ leave $\tau$ unchanged. The strip $\mathcal{S}$ consists of an infinite number of domains $\mathcal{F}_{\gamma}$ each of which can be obtained from $\mathcal{F}$ by the action of an element of $\Gamma, \gamma\left(\rho_{1} \rho_{2}\right)=\left(\begin{array}{cc}a & b \\ \rho_{1} & \rho_{2}\end{array}\right)$ where $\rho_{1}$ and $\rho_{2}$ are relatively prime. The integers $a$ and $b$ can be chosen in such a way that $\operatorname{Im} \tau^{\prime}=\operatorname{Im} \tau / E\left(\tau, \rho_{1}, \rho_{2}\right)$ where we have defined

$$
E\left(\tau, \rho_{1}, \rho_{2}\right) \equiv\left|\rho_{1} \tau+\rho_{2}\right|^{2}
$$

Note that in expression (2.7), if we replace the summation by 1 then what we have is the cosmological constant at one loop for the bosonic string at zero temperature [21] which is invariant under modular transformations. Using these facts we see that

$$
\begin{aligned}
f(\beta) & =-\frac{1}{2}\left(\frac{1}{4 \pi^{2} \alpha^{\prime}}\right)^{-13} \int_{\mathcal{S}} \frac{d^{2} \tau^{\prime}}{\operatorname{Im} \tau^{\prime 2}}\left(\operatorname{Im} \tau^{\prime}\right)^{-12}\left|\eta\left(e^{2 \pi i \tau^{\prime}}\right)\right|^{-48} \sum_{r=1}^{\infty} \exp -\frac{\beta^{2} r^{2}}{4 \pi \alpha^{\prime} \operatorname{Im} \tau^{\prime}} \\
& =-\frac{1}{2}\left(\frac{1}{4 \pi^{2} \alpha^{\prime}}\right)^{-13} \int_{\mathcal{F}} \frac{d^{2} \tau}{\operatorname{Im} \tau^{2}}(\operatorname{Im} \tau)^{-12}\left|\eta\left(e^{2 \pi i \tau}\right)\right|^{-48} \sum_{r_{1}, r_{2}} \exp -\frac{\beta^{2} E\left(\tau, r_{1}, r_{2}\right)}{4 \pi \alpha^{\prime} \operatorname{Im} \tau} .
\end{aligned}
$$

It follows now that the total entropy in the bosonic string at one loop is given by

$$
\begin{aligned}
S=\left(\frac{1}{4 \pi^{2} \alpha^{\prime}}\right)^{-13} 2 \pi A & \int_{0}^{\infty} d z(2 \pi z)^{3} E\left(\tau, r_{1}, r_{2}\right) \times \\
& \times \int_{\mathcal{F}} \frac{d^{2} \tau}{\operatorname{Im} \tau^{2}}(\operatorname{Im} \tau)^{-13}\left|\eta\left(e^{2 \pi i \tau}\right)\right|^{-48} \sum_{r_{1}, r_{2}} \exp -\frac{\pi z^{2} E\left(\tau, r_{1}, r_{2}\right)}{\alpha^{\prime} \operatorname{Im} \tau} .
\end{aligned}
$$

It is easy to check that this expression is modular invariant using the Poisson resummation formula. This means that the restriction of the modular integration to the fundamental domain $\mathcal{F}$ is a consistent procedure. 
Fig. 1: In string theory the modular integration is over the fundamental domain $\mathcal{F}$ and not over the strip $\mathcal{S}$. The entropy is ultraviolet finite because the region of short proper time near $\operatorname{Im} \tau=0$ is excluded by modular invariance. Infrared divergences coming from very large $\operatorname{Im} \tau$ may still be present.

The most important consequence of modular invariance is that the entropy is ultraviolet finite. The troublesome region in the proper time integration that gave rise to the divergence in field theory is excluded in string theory (see fig. 1). By performing the $z$ integral first, we can obtain the entropy as an integral of a density over the fundamental domain.

A striking property of the above formula is that it suffers from a severe infrared divergence. The integral diverges when $z$ is small and $\operatorname{Im} \tau$ is large which corresponds to virtual particles spending a long proper time near the horizon. The interpretation of this divergence is not completely clear to us. One possibility is to render this expression finite by appropriate deformation of the $z$ contour. Note that the expression is modular invariant for an arbitrary contour in the complex $z$ plane. By shifting the contour away from the real axis it is possible to get a finite expression. This is analogous to the situation in statistical mechanics when a partition function has a pole for real $\beta$. One still obtains an exponentially increasing but finite density of states because the proper choice of contour of the inverse Laplace transfom avoids the singularity. Another possibility is that the $z$ integral is defined via a principal value prescription. While both these prescriptions would lead to mathematically well-defined expressions, their physical justification is far from clear. From a physical point of view, it seems much more plausible that the divergence is indicative of a Hagedorn-like transition [15, 22, 23, 16] well-known in string theory. We can 
gain some insight by rewriting the formula (2.14) in a more suggestive form. Using the Poisson resummation formula, it is easy to see that

$$
\sum_{r_{1}, r_{2}} \exp -\frac{\pi z^{2} E\left(\tau, r_{1}, r_{2}\right)}{\alpha^{\prime} \operatorname{Im} \tau}=\frac{1}{2 \pi z} \sqrt{4 \pi \alpha^{\prime} \operatorname{Im} \tau} \sum_{m, n} e^{-2 \pi m n \operatorname{Re} \tau} e^{-\pi \operatorname{Im} \tau\left(\alpha^{\prime} m^{2} / z^{2}+n^{2} z^{2} / \alpha^{\prime}\right)} .
$$

With this equality we make contact with the work of Sathiapalan and Kogan [22]. They observed that the Hagedorn transition can be identified with the appearance of a tachyon that comes from a winding mode in the compactified Euclidean time direction. The radius of the circle in the time direction is $R=z / \sqrt{\alpha^{\prime}}$. In the above formula, the integer $n$ can be identified with the winding number. To look for the tachyonic divergence we look for terms that go as $e^{+\operatorname{Im} \tau}$ at large $\operatorname{Im} \tau$ which then produce a divergence from the modular integration. For example, if we consider the term with $m=0$ and $n= \pm 1$ we find that there is no tachyonic divergence for large $z$ but it first appears at $z=\sqrt{2 \alpha^{\prime}}$. This is only the first tachyonic mode that appears as we lower $z$. At smaller and smaller values of $z$ (i.e., at higher and higher temperatures), more and more winding modes become tachyonic. The exponential growth of the density of states gives rise to poles at these various values of $z$. This means that we must really abandon the thermal ensemble for this region of the $z$ integral. It is worth pointing out in this context that in theories that have the $R \rightarrow \frac{1}{R}$ duality, like the heterotic string, there is no divergence at $z=0$ but only at intermediate values of $z$.

\section{Discussion}

It is equally easy to compute the one loop entropy in superstring theory, by using the known formulae for superstrings at finite temperature [23,24,16]. Once again we obtain a modular invariant expression for the entropy that is ultraviolet finite. The infrared divergences are still present, and they should be. Superstrings do not have a tachyon in the spectrum to begin with. Nevertheless, they have an exponentially growing density of states at high energy and the corresponding Hagedorn transition. The tachyons that are relevant here have to do with this phase transition and they persist even in the case of superstrings. 
It may appear that we have simply traded an ultraviolet divergence for an infrared divergence without gaining anything. After all, we started with the expression in field theory and rewrote it. However, it should be remembered that our ability to restrict the region of integration to the fundamental domain depended crucially on the very specific spectrum of string theory. It would not be possible for an arbitrary collection of field theories. Moreover, the physical interpretation of these two divergences is profoundly different. An ultraviolet divergence is usually indicative of new degrees of freedom or a plain inconsistency of the theory. For example, the ultraviolet divergences in the Fermi theory of weak interactions are indicative of the existence of massive vector bosons. After adding these new degrees of freedom, the ultraviolet divergences are removed from the more complete theory. In this sense, the ultraviolet divergences are unphysical and are a feature only of the low energy theory. Infrared divergences, on the other hand, contain important physics. In the present context, the infrared divergence is indicative of an instability and possibly a phase transition. It says that we have not treated the tree level answer correctly. Ordinarily, in string theory, the tree level free energy is zero because the sphere partition function vanishes by conformal invariance. Here, a tree level contribution to the entropy will be induced by the latent heat of the phase transition.

All our reasoning so far has been from a Hamiltonian point of view. It would be most interesting to obtain the same results from a Euclidean path intgral [3]. The Euclidean continuation of Rindler spacetime is simply flat space with the angular variable in a plane playing the role of Euclidean time. The periodicity $2 \pi$ of the angular variable is proportional to the inverse temperature $\beta$. In order to compute the entropy we need to take a derivative of the free energy with respect to $\beta$. Consequently we need to understand string propagation on a plane where the periodicity of the angular variable differs from $2 \pi$. This amounts to studying string theory on a cone with some deficit angle [14,5, 25]. As described in [25], the twisted sectors play an important role in the formulation of string theory on a conical orbifold. It seems plausible that the twisted states and the winding modes above are somehow related. Both are necessary in string theory to ensure modular invariance but are difficult to describe in the corresponding field theory.

A path integral derivation is desirable for another reason. In this paper, we derived the entropy by integrating a local density. This is certainly correct at one loop. At higher loops, 
the local entropy density may not be very well-defined because the thermal wavelength at a given position is of the same order as the proper distance from the horizon. We wish to return to a more complete comparison of the two approaches in a subsequent publication [26]. We expect that both these methods for computing the entropy should agree in the end, at least at one loop. The necessity for including the winding modes means that the Euclidean path integral for Rindler spacetime should be regarded as a path integral not over a plane, but over a plane with the origin removed. A particle does not notice the difference between the two because there is an ultraviolet divergence near the origin which has to be regulated. For a string, there are no ultraviolet divergences. However, the string knows that it is moving not on a plane but on a topologically nontrivial space and hence the winding modes must be included. Notice that the variable $z$ above measures the radial distance away from the origin. Each winding mode at some radius $z$ represents a state that has a position dependent mass-squared which gets smaller towards the origin and eventually becomes tachyonic.

At this stage, we would like to mention the various approximations that we have used in this calculation:

1. We have worked in the limit of infinite black hole mass. There will certainly be finite mass corrections to the black hole entropy. At present, we do not know how to include these into our calculation.

2. We have ignored finite mass corrections to the entropy in equation (1.3). These are down by powers of $m \epsilon$ where $m$ is the mass of the quantum field and are unimportant as long as $\epsilon$ is strictly zero. Once we have an infrared divergence, we will have to keep $\epsilon$ small but nonzero. In that case, we will have to include the finite mass corrections at some mass level. It is possible to circumvent this difficulty by using the full heat kernel for massive particle on a cone directly [26]. Moreover, for special values of the Rindler temperature we can compare our results with the conical orbifold [25]. We expect that many of the qualitative features such as the existence of the Hagedorn transition etc., will not be altered.

3. There is a more conceptual question regarding the validity of using the thermal ensemble. A Rindler observer sees a hot thermal bath and the thermal ensemble is not 
well-defined in string theory [16]. Moreover, once we include interactions we also have to worry about the Jeans instability [27]. It is not clear how to properly take these effects into account. However, we are really interested only in the entropy at a special value of the temperature and we hope that at least some of the features of the entropy will be accessible without having to understand all the consequences of the Hagedorn transition. For example, it would be nice to see if the entropy can be rendered finite in the infrared by adding a tree level contribution.

Susskind and Uglum [5] have argued that renormalization of entropy can be absorbed into the renormalization of Newton's constant. Their arguments are based on the analysis of ultraviolet divergences in the low energy field theory. Now consider a theory with enough supersymmetries so that there is no renormalization of Newton's constant at one loop. Then, according to Susskind and Uglum, the black hole entropy is given by the Bekenstein-Hawking formula at tree level and there are no loop corrections. The existence of the tree-level answer itself is not explained by this renormalization procedure. In the picture presented in this paper, the entropy is naively zero at tree level. There are no ultraviolet divergences in the full string theory. Nonetheless, there are infrared divergences and the tree-level entropy will be induced by the Hagedorn transition. In this case, the arguments of Susskind and Uglum imply that the induced tree-level entropy must exactly equal the Bekenstein-Hawking entropy.

If this picture turns out to be correct, we may be able to learn something about the Hagedorn transition from its relation to the black hole entropy and vice versa. The Bekenstein-Hawking formula can thus be viewed as a valuable link to the fundamental degrees of freedom of string theory. The black hole entropy will then have a statistical interpretation and the condensate near the horizon can provide a concrete realization, in the context of string theory, of the phenomenological idea of a "membrane" or a "stretched horizon" 28, 29,30,31. 


\section{ACKNOWLEDGEMENTS}

I would like to thank C. Vafa and F. Wilczek for discussions, and J. Gauntlett, J. Preskill, and L. Susskind for comments on the manuscript. This work was supported in part by the U. S. Department of Energy under Grant No. DE-FG03-92-ER40701.

Note added: After completing this work, I learned of a preprint by J. L. F. Barbón [32] in which the thermal ensemble of free strings outside black holes is discussed with the 'brick wall' horizon regularization of 't Hooft. 


\section{References}

[1] J. D. Bekenstein, Phys. Rev. D7 (1973) 2333; Phys. Rev. D9 (1974) 3292.

[2] S. W. Hawking, Phys. Rev. D14 (1976) 2460; Comm. Math. Phys. 43 (1975) 199; Comm. Math. Phys. 87 (1982) 395.

[3] G. W. Gibbons and S. W. Hawking, Phys. Rev. D15 (1977) 2752;

S. W. Hawking, Phys. Rev. D18 (1978) 1747.

[4] G. 't Hooft, Nucl. Phys. B256 (1985) 727.

[5] L. Susskind and J. Uglum, Black Hole Entropy in Canonical Quantum Gravity and Superstring Theory, Stanford Preprint SU-ITP-94-1 (1994), hep-th/9401070.

[6] M. Srednicki, Phys. Rev. Lett. 71 (1993) 666.

[7] J. S. Dowker, Class. Quant. Gravity 11 (1994) L55, hep-th/9401159.

[8] L. Bombelli, R. Koul, J. Lee and R. Sorkin, Phys. Rev. D34 (1986) 373.

[9] C. G. Callan and F. Wilczek, Phys. Lett. 333B (1994) 55, hep-th/9401072.

[10] D. Kabat and M. J. Strassler, Phys. Lett. 329B (1994) 46, hep-th/9401125.

[11] N. D. Birrell and P. C. Davies, Quantum Fields in Curved Space, Cambridge University Press (1982).

[12] T. M. Fiola, J. Preskill, A. Strominger, S. P. Trivedi, Black Hole Thermodynamics and Information loss in Two Dimensions, Caltech Report CALT-68-1918 (1994), hepth/9403137.

[13] L. Susskind, Phys. Rev. D49 (1994) 6606; Phys. Rev. Lett. 71 (1993) 2367.

[14] L. Susskind, Some Speculations about Black Hole Entropy in String Theory, Rutgers University preprint RU-93-44 (1993), hep-th/9309145.

[15] R. Hagedorn, Nuovo Cimento Suppl. 3 (1965) 147;

E. Alvarez, Phys. Rev. D31 (1985) 418;

B. Sundborg, Nucl. Phys. B254 (1985) 583;

S. H. Tye, Phys. Lett. 158B (1985) 388;

M. J. Bowick and L. C. R. Wijewardhana, Phys. Rev. Lett. 54 (1985) 2485.

[16] J. Atick and E. Witten, Nucl. Phys. B310 (1988) 291.

[17] H.J de Vega and N. Sanchez, Nucl. Phys. B299 (1988) 818; Phys. Rev. D42 (1990) 3969; N. Sanchez, Phys. Lett. B195B (1987) 160.

[18] R. Rohm, Nucl. Phys. B237 (1984) 553.

[19] J. Polchinski, Comm. Math. Phys. 104 (1986) 37.

[20] B. Maclain and B. D. B. Roth, Comm. Math. Phys. 111 (1987) 539.

[21] M. B. Green, J. H. Schwarz and E. Witten, Superstring Theory, vols. I and II, Cambridge University Press (1987).

[22] B. Sathiapalan, Phys. Rev. D35 (1987) 3277;

Ya. I. Kogan JETP Lett. 45 (1987) 709.

[23] K. H. O’brian and C. I. Tan, Phys. Rev. D36 (1987) 477. 
[24] E. Alvarez and M. A. R. Osorio, Phys. Rev. D36 (1987) 1175.

[25] A. Dabholkar, String Theory on a Cone and Black Hole Entropy, Harvard University Preprint HUTP-94-A019 (1994), hep-th/9408098

[26] in preparation.

[27] M. J. Bowick and S. B. Giddings, Nucl. Phys. B325 (1989) 631.

[28] K. S. Thorne, R. H. Price, and D. A. MacDonald, Black Holes: The Membrane Paradigm, Yale University Press (1986), and references therein.

[29] R. S. Hanni and R. Ruffini, Phys. Rev. D8 (1973) 3529;

P. Hajicek, Comm. Math. Phys. 36 (1974) 305;

R. L. Znajek, Mon. Not. Roy. Astron. Soc. 185 (1978) 833.

[30] K. S. Thorne and D. A. MacDonald, Mon. Not. Roy. Astron. Soc. 198 (1982) 339;

W. H. Zurek and K. S. Thorne, Phys. Rev. Lett. 54 (1985) 2171;

R. H. Price and K. S. Thorne, Phys. Rev. D33 (1986) 915.

[31] L. Susskind, L. Thorlacius and J. Uglum, Phys. Rev. D48 (1994) 3743, hep-th/9306069.

[32] J. l. F. Barbón, Remarks on Thermal Strings Outside Black Holes, Princeton University Preprint PUPT-94-1478 (1994), hep-th/9406209. 
This figure "fig1-1.png" is available in "png" format from: http://arxiv.org/ps/hep-th/9409158v3

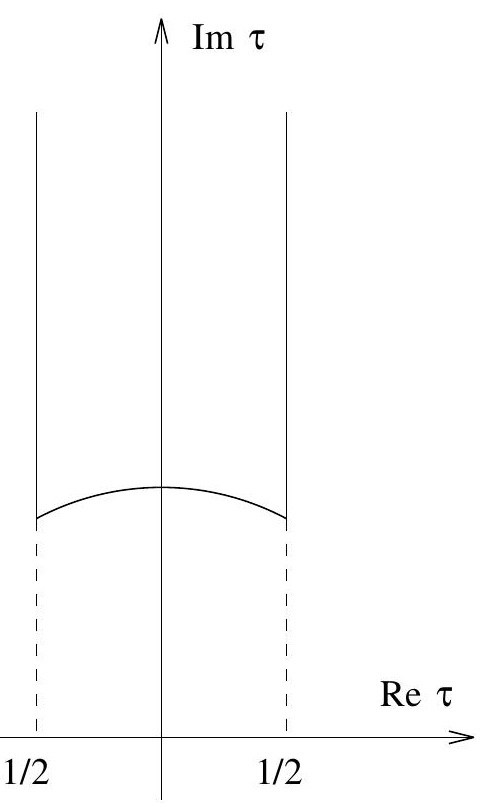

